\title{
Evaluation of the subjective efficacy of nasal surgery
}

\author{
J WU, H R ZANG, T WANG, B ZHOU, J Y YE, Y C LI, D M HAN \\ Department of Otolaryngology Head and Neck Surgery, Beijing Tongren Hospital, Capital Medical University, \\ China
}

\begin{abstract}
Objectives: This study aimed to evaluate subjective symptom changes in obstructive sleep apnoea hypopnea syndrome patients following nasal surgery, and to explore treatment efficacy in improving patient quality of life.

Methods: Patients with nasal blockage accompanied by habitual snoring were stratified into four groups. Their subjective symptoms were evaluated before and after nasal surgery.

Results: There was a significant decrease in the nasal blockage symptom visual analogue scale, Epworth Sleepiness Scale, Snore Outcomes Survey, Spouse/Bed Partners Survey and Sino-Nasal Outcome Test 20 scores for all patients at six months after surgery. The visual analogue scale score for subjective olfactory function was significantly improved in the severe obstructive sleep apnoea hypopnea syndrome patient group.

Conclusion: Nasal surgery can effectively improve the subjective symptoms of patients with simple snoring accompanied by nasal blockage and of patients with obstructive sleep apnoea hypopnea syndrome, thus improving their quality of life.
\end{abstract}

Key words: Nasal Surgical Procedures; Sleep Apnea, Obstructive; Quality of Life

\section{Introduction}

The characteristics of obstructive sleep apnoea hypopnea syndrome (OSAHS) are that the patient's upper airway collapses repeatedly during sleep, resulting in hypopnea or apnoea and leading to daytime sleepiness, decreased work efficiency and depression. These outcomes severely affect patient quality of life (QoL). Multiple risk factors, including upper airway stenosis, obesity, age, sex, heredity and environment, are involved in the disease pathogenesis. ${ }^{1,2}$

Nasal blockage is a common symptom in OSAHS patients. Nasal stenosis can increase nasal airway resistance, weaken the nasopharyngeal reflex, increase mouth breathing during sleep and cause other pathological changes. Nasal surgery can reduce nasal ventilation resistance and improve nasal ventilation function by expanding the effective nasal ventilation volume, alleviating upper airway obstruction during sleep, and reducing the incidence of cardiovascular and cerebrovascular disease, senile metabolic disorders, and other severe diseases. It can significantly improve OSAHS patient QoL. ${ }^{3}$

In this study, patients with OSAHS and simple snoring who complained of nasal blockage and snoring during sleep underwent nasal surgery. Subjective symptoms were compared at baseline and approximately six months after surgery. The efficacy of nasal surgery for improving patients' sleep-related subjective symptoms and QoL was investigated.

\section{Materials and methods}

\section{Participants}

This study included 153 consecutive patients who were hospitalised and treated for nasal blockage accompanied by habitual snoring between January 2011 and December 2014. All patients choose to undergo nasal surgery rather than continuous positive airway pressure treatment. Sinus computed tomography scanning was routinely performed before surgery to identify structural abnormalities of the nasal cavity. Radioallergosorbent tests were also performed to exclude the possibility of allergies. Patients were divided into four groups according to their apnoea hypopnea index (obtained by polysomnography) before surgery: simple snoring group (apnoea hypopnea index $<5), 28$ patients; mild OSAHS group (apnoea hypopnea index $\geq 5$ and $\leq 15$ ), 27 patients; moderate OSAHS group (apnoea hypopnea index $>$ 15 and $\leq 30$ ), 31 patients; and severe OSAHS group (apnoea hypopnea index $>30$ ), 67 patients. 
Patients complaining of nasal blockage accompanied by snoring during night sleep (for at least six months), and those for whom nasal endoscopy or a sinus examination indicated abnormal nasal structures, such as deviated septum, narrow nasal structure, and middle and inferior turbinate hypertrophy, were included. Patients with chronic sinusitis and nasal polyps, a previous history of nasal and sinus surgery, a central sleep breathing disorder, a history of mental illness, or oral deformity and significant tongue hypertrophy, or those taking nasal medications (e.g. steroids, antihistamines, decongestants) or devices were excluded. The study was performed according to the principles stated in the Declaration of Helsinki and was approved by the institutional review board of Beijing Tongren Hospital.

\section{Subjective symptom assessment}

Subjective symptoms were scored six months before and after surgery using the visual analogue scale (VAS), the Epworth Sleepiness Scale, the Sino-Nasal Outcome Test 20 (SNOT-20), the Sleep Outcomes Survey and the Spouse/Bed Partners Survey. ${ }^{4-6}$

\section{Polysomnography}

All patients underwent routine, standard polysomnography (Sandman Elite, Nellcor Puritan Bennett, Ontario, Canada) before surgery. The lowest blood oxygen concentration, average blood oxygen concentration and apnoea hypopnea index were measured. Patients with an apnoea hypopnea index of 5 or more were diagnosed with OSAHS; those with an apnoea hypopnea index of less than 5 were placed into the simple snoring group.

\section{Surgical methods}

All patients had general anaesthesia for nasal surgery. Surgical procedures include septoplasty with three high-tension lines resection, medial displacement and fixation of the middle turbinate, symmetrical bilateral ethmoidectomy and maxillary sinus surgery, and lateral displacement and fixation of the inferior turbinate. At 24-48 hours after surgery, the silicone snorkel was removed from the common nasal meatus and the absorbent cotton and other filling materials were aspirated. One week after surgery, the middle meatus drainage tube was removed and the nasal cavity was washed with normal saline. ${ }^{4}$

\section{Statistical analysis}

A paired $t$-test was used to compare indicators before and after surgery within groups. An independent sample $t$-test was used for between-group comparisons. IBM SPSS Statistics software version 18.0 (Chicago, Illinois, USA) was used for the analysis, and a $p$ value of less than 0.05 was considered statistically significant.

\section{Results}

\section{General patient information}

In all 139 male and 14 female patients aged 18-64 years took part in the study. The mean (standard deviation) apnoea hypopnea index was 2.6 (1.5) for the simple snoring group, 10.1 (2.7) for the mild OSAHS group; 22.1 (3.6) for the moderate OSAHS group and 50.9 (16.6) for the severe OSAHS group. However, only 21 patients agreed to undergo polysomnography monitoring after surgery; the others refused the procedure because they perceived a significant improvement in symptoms. Since repair of the nasal cavity and sinus mucosa takes three to six months, scores were not recorded at earlier time points.

\section{Visual analogue scale score}

After surgery, 148 patients (96.7 per cent) had improved nasal airflow; the average nasal blockage VAS score decreased significantly for each patient group. Three of the groups showed no significant change in olfactory function $(p>0.05)$, but patients with severe OSAHS showed a significant improvement (Table I).

\begin{tabular}{|c|c|c|c|c|c|}
\hline NASAL & $\mathrm{GE} A$ & $\begin{array}{r}\text { TA } \\
\text { TORY FUNCTI }\end{array}$ & SCORES BEF & AFTER SUR & \\
\hline \multirow[t]{2}{*}{ Survey instrument } & \multirow[t]{2}{*}{ Group } & \multicolumn{2}{|c|}{ Score* } & \multirow[t]{2}{*}{ Decrease ratio } & \multirow[t]{2}{*}{$p$ value } \\
\hline & & Before surgery & After surgery & & \\
\hline \multirow[t]{4}{*}{ Left nasal blockage } & A & $5.81 \pm 2.89$ & $2.46 \pm 2.42$ & 57.7 & $\dagger$ \\
\hline & B & $5.70 \pm 3.30$ & $1.62 \pm 1.76$ & 71.6 & $\dagger$ \\
\hline & $\mathrm{C}$ & $5.53 \pm 3.25$ & $1.59 \pm 1.66$ & 71.2 & $\dagger$ \\
\hline & $\mathrm{D}$ & $5.10 \pm 3.22$ & $1.88 \pm 2.23$ & 63.1 & $\dagger$ \\
\hline \multirow{4}{*}{ Right nasal blockage } & A & $5.35 \pm 3.09$ & $2.23 \pm 2.47$ & 58.3 & $\dagger$ \\
\hline & $\mathrm{B}$ & $4.91 \pm 3.17$ & $1.35 \pm 1.40$ & 72.5 & $\dagger$ \\
\hline & $\mathrm{C}$ & $5.42 \pm 3.41$ & $1.30 \pm 1.56$ & 76.0 & $\dagger$ \\
\hline & $\mathrm{D}$ & $5.47 \pm 3.31$ & $1.75 \pm 1.75$ & 68.0 & $\dagger$ \\
\hline \multirow{4}{*}{ Olfactory functions } & A & $2.30 \pm 3.19$ & $1.69 \pm 2.43$ & 26.5 & 0.310 \\
\hline & $\mathrm{B}$ & $2.63 \pm 2.85$ & $1.55 \pm 2.11$ & 41.6 & 0.051 \\
\hline & $\mathrm{C}$ & $3.10 \pm 3.11$ & $1.80 \pm 2.87$ & 41.9 & 0.053 \\
\hline & D & $2.60 \pm 3.32$ & $1.27 \pm 1.93$ & 51.2 & \\
\hline
\end{tabular}

${ }^{*}$ Data are means \pm standard deviation. ${ }^{\dagger} p<0.001 . \mathrm{VAS}=$ visual analogue scale; $\mathrm{A}=$ simple snoring group; $\mathrm{B}=$ mild OSAHS group; $\mathrm{C}=$ moderate OSAHS group; $\mathrm{D}=$ severe OSAHS group 
Sleep Outcomes Survey, Spouse/Bed Partner Survey and Epworth Sleepiness Scale scores

Six months after surgery, the Sleep Outcomes Survey and Spouse/Bed Partners Survey scores had decreased significantly for each group ( $p<0.01$; Figure 1$)$; patients with moderate OSAHS had the largest reduction in score (Sleep Outcomes Survey, 32.15 per cent; Spouse/Bed Partners Survey, 36.18 per cent). For all groups, the degree of improvement was greater for the Spouse/Bed Partners Survey score than for the Sleep Outcomes Survey score.

The Epworth Sleepiness Scale score for each group also decreased significantly after surgery $(p<0.01$; Figure 1): scores for patients in the simple snoring and severe OSAHS groups decreased by approximately 30 per cent and those for patients in the mild and moderate OSAHS groups decreased by approximately 40 per cent. Patients with moderate OSAHS had the greatest decrease in score (45.65 per cent).

\section{Sino-Nasal Outcome Test 20 score}

The SNOT-20 scores related to nose and related symptoms, sleep symptoms, activity, and emotion were significantly lower following surgery for all groups.
Scores in the dimensions of nasal and related symptoms improved by more than 60 per cent in all four groups. Scores in the sleep symptom dimension decreased by more than 50 per cent in all groups, and by nearly 70 per cent in the moderate OSAHS group. For all groups, improvements in the activity-related and emotion-related dimensions (which are closely associated with psychological and social function) also improved by more than 50 per cent. The total SNOT20 score for all groups also decreased significantly (Table II).

\section{Surgical complications}

None of the patients in this study experienced a severe surgical complication. Some patients had transient mucosal oedema after surgery, although all symptoms significantly improved after conservative treatment.

\section{Discussion}

According to the World Health Organization, healthrelated QoL is an individual's perception of living conditions related to their goals, expectations, standards and concerns in different cultural and value systems, including physiological health, psychological status,
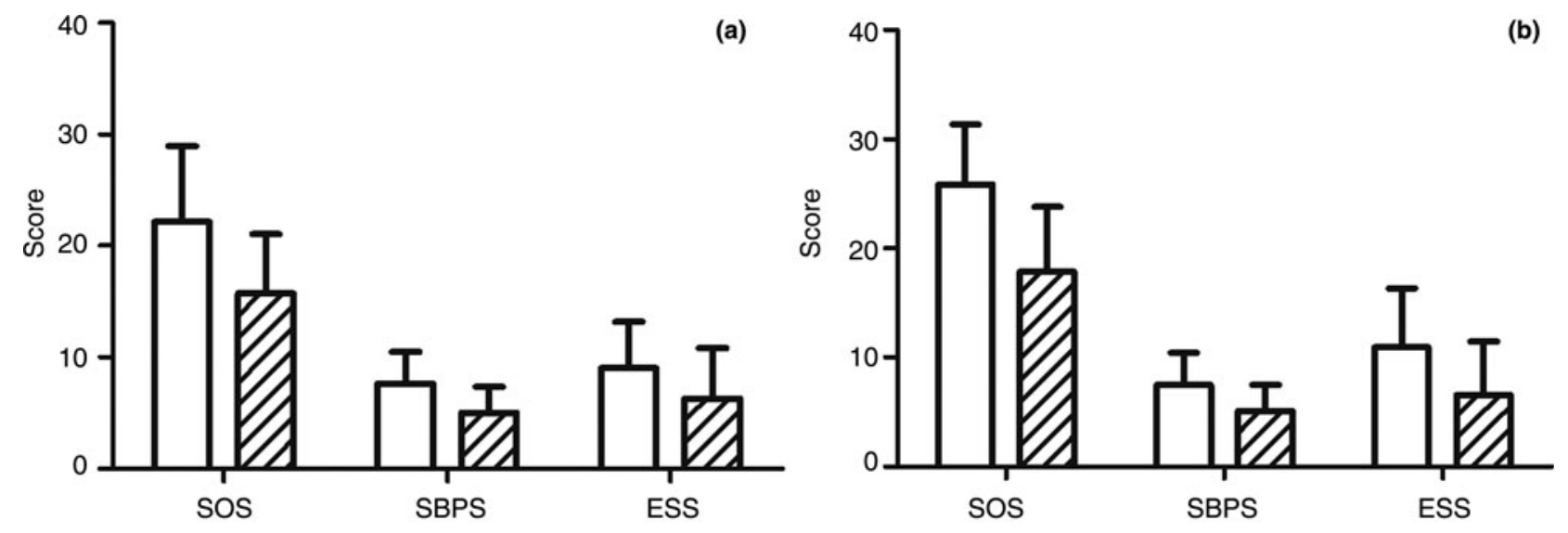

(c)
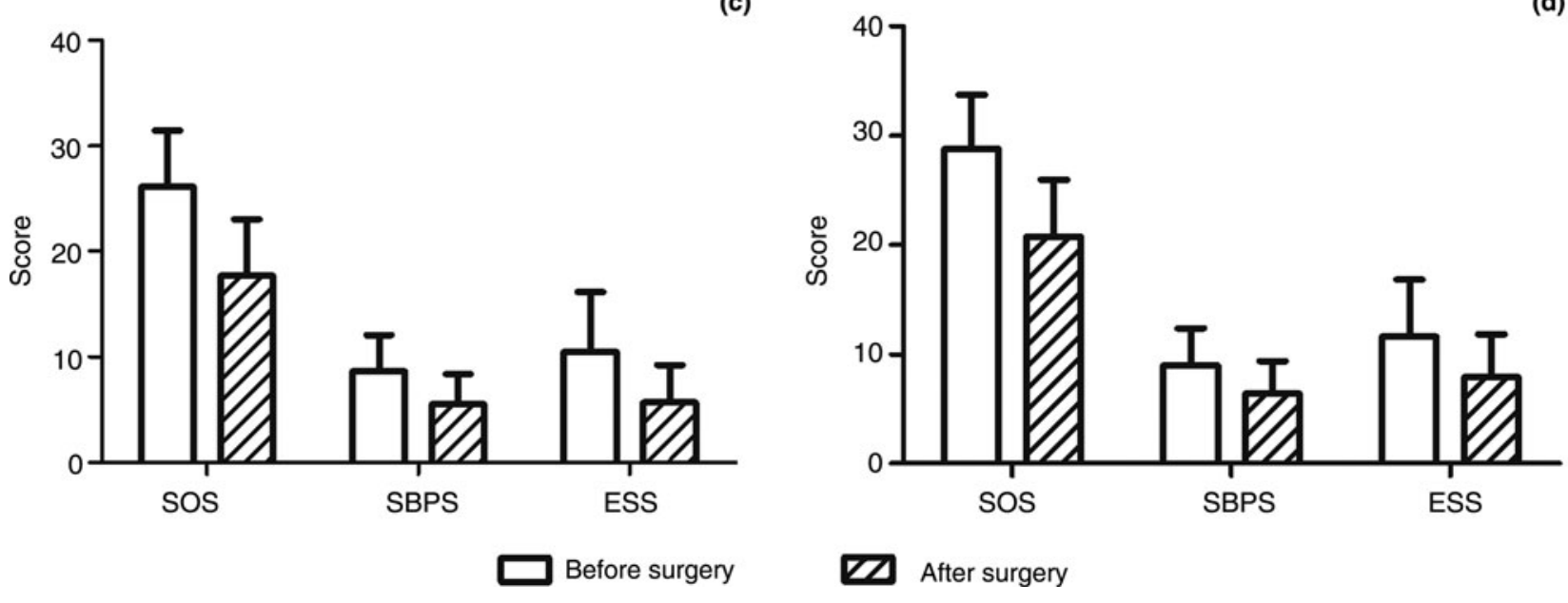

FIG. 1

Sleep Outcomes Survey, Spouse/Bed Partners Survey and Epworth Sleepiness Scale scores before and after surgery in the (a) simple snoring, (b) mild OSAHS, (c) moderate OSAHS and (d) severe OSAHS groups. All scores were significantly improved after surgery $(p<0.001)$. ESS = Epworth Sleepiness Scale; SBPS = Spouse/Bed Partners Survey; SOS = Sleep Outcomes Survey 


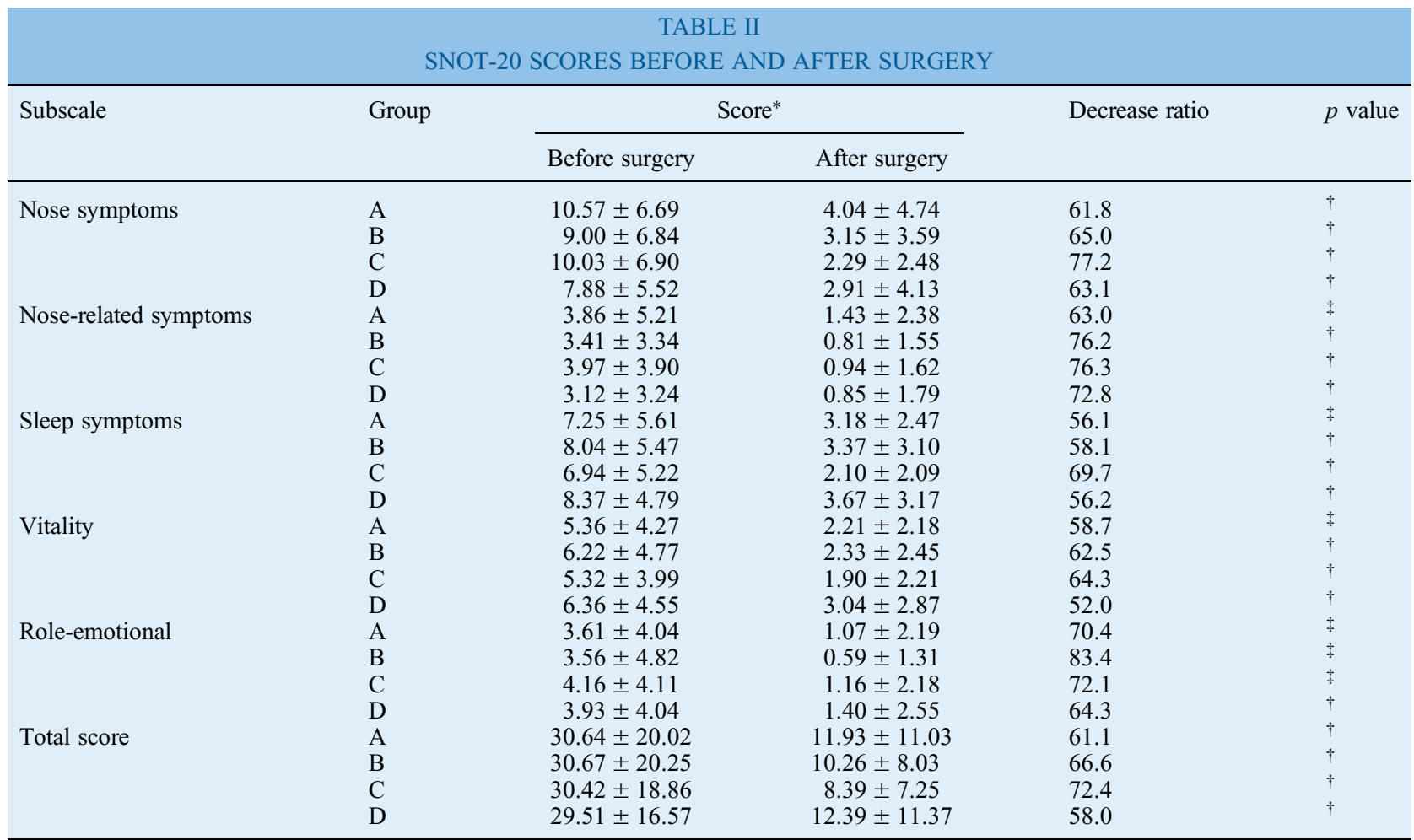

${ }^{*}$ Data are means \pm standard deviation. ${ }^{\dagger} p<0.001 .{ }^{\star} p<0.05$. SNOT-20, Sino-Nasal Outcome Test $20 ; \mathrm{A}=$ simple snoring group; $\mathrm{B}=$ mild OSAHS group; $\mathrm{C}=$ moderate OSAHS group; $\mathrm{D}=$ severe OSAHS group

independence ability, social relationships, personal beliefs and relationship with the surrounding environment. ${ }^{7}$ The biopsychosocial medical model is becoming increasingly popular. Therefore, clinicians should consider patient perception on the impact of disease on health and life as an effective method of assessing QoL. The subjective evaluation of nasal blockage and sleep snoring symptoms by patients with simple snoring or OSAHS more accurately reflects changes in their physiological, psychological and social function before and after nasal surgery, thus providing a better evaluation of their QoL. In addition, subjective symptom evaluation can help physicians in clinical practice to determine which factors have the greatest influence on patient QoL, thus providing a basis for patient prognosis after surgery.

Under normal conditions, nasal resistance accounts for approximately 50 per cent of the respiratory tract's total resistance. Nasal obstructive diseases increases nasal resistance and can increase the total resistance of the upper respiratory tract, increase the pharyngeal negative pressure gradient and cause the collapse of aggravated pharyngeal soft tissue. The latter can increase the probability of sleep apnoea and aggravate disease severity in OSAHS patients. It thus severely affects their physiological and psychological activities in their social lives. ${ }^{8-12}$ Some researchers consider that nasal obstruction plays a larger role in the pathophysiological mechanism of sleep apnoea hypopnea only in mild OSAHS patients, and that it may have less effect in severe OSAHS patients. ${ }^{9}$
Therefore, this study evaluated the effect of nasal surgery on relieving upper airway origin obstruction and improving OSAHS via reducing nasal resistance in patient groups stratified according to OSAHS severity (according to the apnoea hypopnea index). Data from the simple snoring group (apnoea hypopnea index $<5$ ) were included for comparison.

Snoring is generated by vibration of the soft palate, the pharynx, the epiglottis, the tongue and other pharyngeal tissue structures. Snoring during sleep is common in OSAHS patients. Airflow restriction resulting from the decreased cross-sectional area of the upper airway causes vibration. ${ }^{13}$ Some studies have shown that snoring during sleep can cause sleep structure disorders and sleep fragmentation, resulting in decreased sleep quality, daytime sleepiness, difficulty in concentrating, and reduced work efficiency and QoL. ${ }^{14}$ Moreover, noisy snoring can affect a spouse's sleep quality. The combined use of the Sleep Outcomes Survey and Spouse/Bed Partners Survey rating scale provides a more complete evaluation of the patients' snoring duration, loudness and frequency; it can also adequately evaluate both a spouse's intuitive feeling of the patient's snoring and the impact of snoring on the spouse's QoL. Li et al. found that after nasal surgery, the Spouse/Bed Partners Survey score of spouses improved by 50.4 per cent, whereas the improvement in patients' Sleep Outcomes Survey snoring scores was slightly lower (43.1 per cent). ${ }^{12} \mathrm{~A}$ similar result was obtained in the present study: for patients in all OSAHS groups, there was a greater 
decrease in the Spouse/Bed Partners Survey score after nasal cavity ventilation expansion surgery (Figure 1). In addition, the performance of the Sleep Outcomes Survey and the Spouse/Bed Partners Survey for simple snoring patients also deserves clinical attention. For the Sleep Outcomes Survey score, the self-evaluation of most patients is likely to contain errors. A more accurate understanding of the effect of snoring on the spouse's sleep quality and QoL is provided by the Spouse/Bed Partners Survey score. Therefore, nasal surgery effectively relieves patients' snoring symptoms during sleep. The improvement in snoring after surgery means that snoring has a much lower impact on the spouse. Therefore, nasal surgery can improve the sleep quality of both patients and their spouses.

Another common symptom of OSAHS patients is daytime sleepiness. Patients frequently experience different degrees of irresistible sleepiness regardless of occasion, which causes significant emotional distress or a decreased ability to function in both work and social situations. Changes in cognitive function can also be manifested as memory loss, a decline in thinking ability and a reduced ability to learn new knowledge, which severely affect patients' work efficiency and social function. ${ }^{15}$ Currently, the Epworth Sleepiness Scale, designed by Professor Johns, is commonly used worldwide to evaluate the degree of daytime sleepiness. ${ }^{4}$ It has a simple, rational design and it is easy to score and operate. By comparing 100 OSAHS patients with 61 Chinese people without OSAHS, Chung found that the Epworth Sleepiness Scale score was significantly higher for OSAHS patients than for non-OSAHS patients $(13.2 \pm 4.7 \mathrm{vs}$ $7.5 \pm 3.09) .{ }^{16}$ Other studies have confirmed that snoring severity correlates positively with sleep fragmentation, and that sleep fragmentation is an important cause of daytime sleepiness. ${ }^{17}$ Recently, a meta-analysis of eight publications based on the Epworth Sleepiness Scale found that the Epworth Sleepiness Scale score for OSAHS patients decreased significantly after nasal surgery $(10.6 \pm 3.9 v s \quad 7.1 \pm$ $3.7, p<0.001)$, indicating that nasal surgery can improve daytime sleepiness symptoms. ${ }^{18}$ In the present study, the Epworth Sleepiness Scale score before surgery was slightly lower for simple snoring patients than for the other three groups. The Epworth Sleepiness Scale score increased with increasing OSAHS severity (Figure 1). After surgery, the score for patients in each group decreased to a normal level (i.e. $<8$ points), but the decrease was greatest for patients in the moderate OSAHS group (45.65 per cent). The decrease in Sleep Outcomes Survey and Spouse/Bed Partners Survey scores were also greatest for patients in this group. This finding might relate to OSAHS severity because anatomical stenosis of the upper airway, especially pharyngeal stenosis, is less severe in moderate than in severe OSAHS patients. Although nasal cavity stenosis was improved in severe OSAHS patients after surgery, the pharyngeal plane continued to be severely obstructed. In mild OSAHS patients, the major obstructive sites were primarily located in the nasal cavity, and nasal stenosis was less severe in these patients than moderate OSAHS patients. Therefore, patients in the moderate OSAHS group had the best improvement in effective nasal ventilation volume following nasal surgery: compared with patients in the mild and severe OSAHS groups, they had the most significant improvements in snoring strength and sleepiness symptoms. For each group, relief from sleepiness after surgery was related to the removal of upper airway origin obstruction, reduced upper airway total resistance, reduced breathing through the mouth, a reduced pharyngeal lumen negative pressure gradient, increased effective ventilation volume per minute during sleep, increased blood oxygen metabolic level during the night, improved sleep structure and a decreased frequency of wakening.

The SNOT-20 score scale is a revised version of the Rhinosinusitis Outcome Measure 31 scale that better reflects the nasal, sleep and emotional conditions of patients. ${ }^{6}$ However, the SNOT-20 score does not evaluate olfactory function; therefore, this study included the olfactory function dimension of the VAS. Moreover, by combining the SNOT-20 score with the nasal blockage VAS evaluation, a better understanding of patients' subjective feelings about nasal resistance before and after surgery can be obtained. In this study, the nasal blockage VAS score and SNOT-20 middle nasal symptom, nose-related symptom, sleep disorder, activity, and emotion scores of patients in the simple snoring group improved significantly after surgery. Moreover, all indicators decreased significantly after surgery for patients in all OSAHS groups (except the olfactory function VAS score for mild and moderate OSAHS patients). This finding was corroborated by the Epworth Sleepiness Scale, Sleep Outcomes Survey and Spouse/Bed Partners Survey scores. The following mechanism is proposed to be responsible for this effect. Firstly, nasal surgery opens the bilateral middle nasal meatus, enlarges the natural openings of the maxillary sinus, opens the anterior and posterior ethmoidal sinuses, selectively opens the frontal recess and enlarges the aperture of the frontal sinus, and opens the aperture of the sphenoidal sinus, which aids the smooth drainage of sinus secretions, thus relieving nose blowing and other nasal symptoms. Secondly, the simultaneous opening of sinuses removes the anatomical cause of negative pressure headache, thus relieving headache, dizziness and other symptoms. Thirdly, decreased ventilation resistance in the upper airway reduces the frequency of mouth breathing during sleep, improves the degree of oropharyngeal cavity collapse and/or stenosis and negative pressure in the downstream pharyngeal airway, reduces the degree of pharyngeal collapse stenosis, reduces the number of night-time hypopnea and apnoea episodes, 
and improves related sleep and emotional symptoms. Fourthly, the improvement in nasal and sleep-related symptoms leads directly to improved emotional and activity indicators that are closely related to the psychological and social function of patients. The olfactory function of severe OSAHS patients is significantly improved, possibly because these patients generally have a more severe nasal blockage, greater probability of an abnormal nasal structure and increased chronic mucosal inflammation, and more severe stenosis of the olfactory cleft, along with a reduction or complete absence of olfactory particles in the airflow reaching the olfactory area. While expanding the effective ventilation volume, nasal cavity ventilation expansion surgery opens the bilateral middle nasal meatus symmetrically, which slows airflow into the middle nasal meatus. Moreover, after superior meatus opening, air can enter the olfactory cleft through the vortex mechanism, thereby improving olfactory function. In addition, as well as correcting an abnormal nasal anatomical structure, nasal cavity ventilation expansion surgery aims to maximally protect nasal mucosa and minimally damage nasal physiological function. ${ }^{7}$ It can therefore improve nasal and upper airway physiological function and psychological status, thus improving patient QoL.

- Obstructive sleep apnoea hypopnea syndrome severely affects patient quality of life

- Patients can experience daytime sleepiness, decreased work efficiency and depression

- Nasal surgery can reduce nasal ventilation resistance

- It can also improve sleep-related subjective symptoms in patients with simple snoring or obstructive sleep apnoea hypopnea syndrome who complain of nasal blockage

Using computational fluid dynamics, Zang et al. recently confirmed that increased nasal resistance in OSAHS patients increases the pharyngeal negative pressure gradient, which increases pharyngeal airflow speed. ${ }^{19}$ These researchers found that nasal surgery can relieve the degree of nasal blockage, daytime sleepiness and snoring during sleep for OSAHS patients and can reduce the apnoea hypopnea index $(12.1 \pm 3.7$ vs $7.1 \pm 2.6, p<0.05)$ and sleep-wake indicators for mild OSAHS patients. Surgery can also increase minimal blood oxygen saturation during night-time sleep. However, there was no significant improvement in the polysomnography indicator for moderate and severe OSAHS patients. The present study similarly found a significant improvement in the subjective symptom scores for OSAHS patients in each group. There might be several reasons for differences between the subjective and objective symptoms of OSAHS patients following nasal surgery. Firstly, there are vast differences in the extent of nasal surgery for treating OSAHS, surgical methods vary and the eligibility requirements for surgery differ. Reports include only simple deviated septum surgery, with or without partial resection of the inferior turbinate, nasal polyp resection, and sinus open surgery. The different treatments and surgical methods affect overall evaluation of the efficacy of nasal surgery for OSAHS. Secondly, the degree of pharyngeal stenosis is a key factor affecting the efficacy of nasal surgery in treating OSAHS. For example, obesity, Friedman score and Mallampati classification also affect nasal surgery efficacy. $^{20}$ Thirdly, patient selection criteria and follow-up times differ among studies. Although the present study grouped patients according to the apnoea hypopnea index, the sample size of each group was small. The duration of follow up was also a limiting factor. Fourth, the pathophysiological mechanism of OSAHS involves multiple systems at multiple levels, and many factors affect its incidence and prognosis. However, it is even more important to study patient QoL. Multicentre collaborative studies with a larger sample size and longer follow up are required to provide a more scientific and comprehensive evaluation of disease severity and treatment efficacy.

\section{Conclusion}

Through subjective symptom evaluation, this study confirmed that nasal surgery can effectively improve subjective sleep-related symptoms in OSAHS and simple snoring patients who complain of nasal blockage accompanied by sleep snoring. In addition, surgery can improve patient QoL. However, the study did not include a grading system based on nasal pathology; therefore, its findings should be used with caution.

\section{Acknowledgements}

This work was supported by Beijing Natural Science Foundation of China (grant number 7100002).

References

1 Nagai T, Imamura M, Iwasaki Y, Mori M. Obstructive sleep apnea syndrome accompanied by diabetes mellitus. J Med 2003;34:23-30

2 Yaggi HK, Concato J, Kernan WN, Lichtman JH, Brass LM, Mohsenin V. Obstructive sleep apnea as a risk factor for stroke and death. $N$ Engl J Med 2005;353:2034-41

3 Han D, Zhang L. Nasal cavity ventilation expansion techniques. Acta Otolaryngol 2011;131:1244-8

4 Johns MW. A new method for measuring daytime sleepiness: the Epworth sleepiness scale. Sleep 1991;14:540-5

5 Gliklich RE, Wang PC. Validation of the snore outcomes survey for patients with sleep-disordered breathing. Arch Otolaryngol Head Neck Surg 2002;128:819-24

6 Piccirillo JF, Merritt MG, Richards ML. Psychometric and clinimetric validity of the 20 -item Sino-Nasal Outcome Test (SNOT20). Otolaryngol Head Neck Surg 2002;126:41-7

7 World Health Organization Division of Mental Health and Prevention of Substance Abuse. WHOQOL User Manual: Programme on Mental Health. Geneva: World Health Organization, 1998

8 Löth S, Petruson B, Wirén L, Wilhelmsen L. Better quality of life when nasal breathing of snoring men is improved at night. Arch Otolaryngol Head Neck Surg 1999;125:64-7 
9 Virkkula P, Bachour A, Hytönen M, Salmi T, Malmberg H, Hurmerinta $\mathrm{K}$ et al. Snoring is not relieved by nasal surgery despite improvement in nasal resistance. Chest 2006;129:81-7

10 Kim ST, Choi JH, Jeon HG, Cha HE, Kim DY, Chung YS. Polysomnographic effects of nasal surgery for snoring and obstructive sleep apnea. Acta Otolaryngol 2004;124:297-300

11 Georgalas C. The role of the nose in snoring and obstructive sleep apnoea: an update. Eur Arch Otorhinolaryngol 2011; 268:1365-73

12 Li HY, Lin Y, Chen NH, Lee LA, Fang TJ, Wang PC. Improvement in quality of life after nasal surgery alone for patients with obstructive sleep apnea and nasal obstruction. Arch Otolaryngol Head Neck Surg 2008;134:429-33

13 Fiz JA, Abad J, Jané R, Riera M, Mañanas MA, Caminal P et al. Acoustic analysis of snoring sound in patients with simple snoring and obstructive sleep apnoea. Eur Respir $J$ 1996;9: 2365-70

14 Olsen KD, Kerne B. Nasal influences on snoring and obstructive sleep apnea. Mayo Clin Proc1990;65:1095-105

15 Guilleminault C, Partinen M, Quera-Salva MA, Hayes B, Dement WC, Nino-Murcia G. Determinants of daytime sleepiness in obstructive sleep apnea. Chest 1988;94:32-7

16 Chung KF. Use of the Epworth Sleepiness Scale in Chinese patients with obstructive sleep apnea and normal hospital employees. J Psychosom Res 2000;49:367-72

17 Kapur VK, Baldwin CM, Resnick HE, Gottlieb DJ, Nieto FJ. Sleepiness in patients with moderate to severe sleep-disordered breathing. Sleep 2005;28:472-7
18 Li HY, Wang PC, Chen YP, Lee LA, Fang TJ, Lin HC. Critical appraisal and meta-analysis of nasal surgery for obstructive sleep apnea. Am J Rhinol Allergy 2011;25:45-9

19 Zang HR, Li LF, Zhou B, Li YC, Wang T, Han DM. Pharyngeal aerodynamic characteristics of obstructive sleep apnea/ hypopnea syndrome patients. Chin Med J (Engl) 2012;125: 3039-43

20 Koutsourelakis I, Georgoulopoulos G, Perraki E, Vagiakis E, Roussos C, Zakynthinos SG. Randomised trial of nasal surgery for fixed nasal obstruction in obstructive sleep apnoea. Eur Respir J 2008;31:110-17

Address for correspondence:

Dr D M Han,

1 Dong Jiao Min Xiang,

Eastern District,

Beijing 100730, China

Fax: +86 01058268341

E-mail: handemin_ent@163.com

Dr D M Han takes responsibility for the integrity of the content of the paper

Competing interests: None declared 